\title{
A Geometric-Functional-Based Image Segmentation and Inpainting
}

\author{
Vladimir Kluzner ${ }^{1}$, Gershon Wolansky ${ }^{2}$, and Yehoshua Y. Zeevi ${ }^{3}$ \\ ${ }^{1}$ Mathematics Department, Technion \\ mavladim@tx.technion.ac.il \\ 2 Mathematics Department, Technion, gershonw \\ @math.technion.ac.il \\ 3 Department of Electrical Engineering, Technion \\ zeevi@ee.technion.ac.il
}

\begin{abstract}
The Mumford-Shah functional minimization, and related algorithms for image segmentation, involve a tradeoff between a twodimensional image structure and one-dimensional parametric curves (contours) that surround objects or distinct regions in the image.

We propose an alternative functional that is independent of parameterization; it is a geometric functional which is given in terms of the geometry of surfaces representing the data and image in a feature space. The $\Gamma$-convergence technique is combined with the minimal surface theory in order to yield a global generalization of the Mumford-Shah segmentation functional.
\end{abstract}

\section{Introduction}

Let $g(x, y)$ be the intensity of the light signal impinging on a planar image domain $B$ at a point $(x, y)$. The image $g(x, y)$ is expected to be discontinuous along the edges of the objects. The definition of the segmentation depends on whether one approaches the problem at the level of the image as a whole or, alternatively, considers the image as a collection of edge fragments. In the first case it is natural to consider the partitioning of the image into smaller structures. In the second case it becomes more natural to consider the problem of grouping of elements into larger structures. In both cases, the following questions arise: (i) What exactly is the goal of the segmentation process? (ii) Is segmentation feasible? These questions are important for understanding of the above process. Without a clear conception of the task and its requirements no satisfactory progress in this area can be made.

The above dichotomy into local vs. global, and related heuristic approaches, were later circumvented by the variational approach to segmentation, adopted by us and further developed in this paper.

Morel and Solimini [6] showed that any heuristic segmentation method may be translated into a variational one. Variational formulations summarize all criteria concerning a set of edges $K$ in a single real-valued functional $F(K)$, i.e. to any set of edges or "segmentation" $K$ is associated a value $F(K)$ which states how 
"good" the segmentation is. $F$ is defined in such a way that $F(K)$ is better the lower it is. This fact lends itself to an obvious comparison principle: given two segmentations, it must determine which is better. In other words, a variational method implicitly orders all segmentations. Any order can be quantified by $F(K)$ such that $F(K)>F\left(K^{\prime}\right)$ if $K^{\prime}$ is better than $K$.

The present study merges the $\Gamma$-convergence technique (see [9]) and the minimal surface theory (see 3]) to yield a global generalization of the Mumford-Shah segmentation functional. We then apply the functional model to segmentation and inpainting problems. Proofs of theorems, propositions and lemmas are presented elsewhere [5].

\section{Measure-Based Metric Function}

Mumford and Shah [7] proposed the functional

$$
F_{M S}(u, K)=\int_{B \backslash K}|\nabla u|^{2}+|K|+\alpha \int_{B}|u-g|^{2},
$$

where $K \subset B$ is a one dimensional set which represents the edges, and $|K|$ is the length of this set (understood as the one-dimensional Hausdorff measure of $K)$. A minimizer $\{K, u\}$ of $F_{M S}$ thus produces the required image $u$ as well as the edges $K$.

We propose an alternative functional which is independent of parameterization, i.e a geometric functional which may be given in terms of the geometry of surfaces representing the data and image in the feature space. Considering the image $u$ as a two-dimensional surface, we shall replace the first two terms of (1) by the area of this surface. This allows sharp discontinuities (edges) of the image in the form of surface folding. This idea is not new (see, e.g, [11]). However, to make the third term of (1) fit the geometrical description, we must replace it by another metric $\mathcal{D}(g, u)$ representing the distance between the two surfaces. Note that the last term connects locally the image $u(b)$ for each $b \in B$ to the data $g$ at the same point $b$. It implicitly assumes that there is a one-to-one correspondence between points of the data to points of the image.

The proposed modified functional allows us to replace the deterministic data $g$ by a random one. For this we replace $g(b)$ by a measure $\mu_{b}(d y) d b$, where $y$ is a parameterization of the feature fiber. Further, to different pixels there may correspond different amount of data, so $\int \mu_{b}(d y) d b \leq 1$ for each pixel $b$ where strong inequality and even zero value may not be excluded for some of the pixels. Our objective is, essentially, to define a metric $\mathcal{D}(\mu, U)$.

Let us define first the feature space. Let $\mathbf{Y}$ be a set representing the possible data at a single pixel $b \in B$. It may be considered as a real number (the brightness) or a vector (if several color channels are present or, for example, a Gabor-wavelet filter). We shall assume here that $\mathbf{Y}=\mathbb{R}^{m}$. The feature space is, then, defined as the cylinder $E:=B \times \mathbf{Y} \subset \mathbb{R}^{m+2}$. The data $g$ is represented as a measure $\mu$ supported in $E$. 
To define an image $u$, let us consider the two-dimensional unit disc $S:\{|s|<$ $1\}$ diffeomorphic to $B$. We represent the image as a mapping $U: S \rightarrow E$ such that $U(\partial S) \subset \mathbf{C}:=\partial B \times \mathbf{Y}$ is projected onto $\partial B$. Coordinates in the feature space are described by $z:=\{b, y\}$.

The feature space $E$ is endowed with a metric

$$
d z^{2}=\gamma d b^{2}+d y^{2}:=\sum_{i, j=1}^{m+2} h_{i, j} d z_{i} d z_{j}
$$

where $d b^{2}=d b_{1}^{2}+d b_{2}^{2}, d y^{2}=\sum_{i=1}^{m} d y_{i}^{2}$ are the Euclidean metric in $\mathbb{R}^{2}$ and $\mathbb{R}^{m}$, respectively, while $\gamma>0$ represents the relation between the geometric (pixeldomain) and feature metrics. With this setting, the embedding $U(S)$ into $E$ is endowed with the induced metric

$$
\Gamma_{i, j}(U)=U_{s_{i}}^{T} h U_{s_{j}} \quad \text { for } \quad i, j=1,2 .
$$

Setting $|\Gamma(U)|:=\left|\Gamma_{1,1}(U) \Gamma_{2,2}(U)-\Gamma_{1,2}^{2}(U)\right|^{1 / 2}$, the surface area of $U(S)$ is

$$
A(U)=\int_{S}|\Gamma(U)(s)| d s .
$$

Let us replace the first two terms of (1) by (2), and the last term by a distance between the embedded surface $U(S)$ and the data measure $\mu$ :

$$
F(U)=A(U)+\alpha \mathcal{D}^{2}(\mu, U) .
$$

By our convention, the distance $\mathcal{D}$ should only depend on the image $U(S)$ and not on a particular parameterization. With this assumption, we replace $A(U)$ by the quadratic form

$$
\widetilde{A}(U)=\frac{1}{2} \int_{S} \operatorname{Tr}\left(\nabla U(s)^{T} h \nabla U(s)\right) d s,
$$

where $\operatorname{Tr}(\cdot)$ is a trace of a given matrix. Note that

$$
\widetilde{A}(U) \geq A(U)
$$

which reduces to an equality if the parameterization of $U$ is conformal,

$$
\Gamma_{1,1}(U)=\Gamma_{2,2}(U), \quad \Gamma_{1,2}(U)=\Gamma_{2,1}(U)=0 .
$$

We replace $F(U)$ by

$$
\widetilde{F}(U)=\widetilde{A}(U)+\alpha \mathcal{D}^{2}(\mu, U)
$$

and obtain by (5)

$$
\widetilde{F}(U) \geq F(U),
$$

and equality if the embedded $U$ is a conformal mapping from $S$ to $E$. 
For parametric representation of an image used in this section we cannot exclude the possibility that the optimal image is folded over the pixel space $B$. If this is the case, then some pixels of the image over $B$ may have multiple values.

Our first object is to define a distance between two positive measures $\mu$ and $\nu$ supported in $E$, where $\nu$ represents the image and $\mu$ the data. Let

$$
d(A, B)=\sqrt{\sup _{v \in B} \inf _{u \in A}|u-v|^{2}}
$$

and

$$
D(\mu, \nu)=d(\operatorname{supp}(\mu), \operatorname{supp}(\nu))
$$

Lemma 1. For fixed, compactly supported $\mu$ in $E, D(\mu, \nu)$ is lower semicontinuous with respect to $\nu$ under the topology of weak $C^{*}$ convergence.

We now replace the measure $\nu$ by a mapping $U$. Let $l$ be a measure on the parameter space $S$ (say, the uniform Lebesgue measure). We denote the measure $\nu$ associated with an embedding $U \in \mathbb{H}^{1}(S, E)$ by $\nu_{U}$ and define it by the pullback $\nu_{U}(\sigma)=l\left(U^{-1}(\sigma \cap U(S))\right)$ for every Borel measurable set $\sigma \subset E$. In terms of its action on a test function $\phi \in C_{0}(E)$ the above measure is defined in the following way:

$$
\left\langle\nu_{U}, \phi\right\rangle=\int_{S} \phi(U(s)) d s .
$$

We conclude that (8) extends to any $U \in \mathbb{H}^{1}(S, E)$ and define

$$
\mathcal{D}(\mu, U):=D\left(\mu, \nu_{U}\right)
$$

From Lemma 1 we obtain:

Lemma 2. The metric $\mathcal{D}(\mu, U)$ is weakly lower semi-continuous with respect to $U$ in the $\mathbb{H}^{1}$ topology.

We consider now the existence of the minimizer of functional $\widetilde{F}$. Let $U: S \rightarrow E$ be written in the form $U:=\left\{U^{B}, U^{\mathbf{Y}}\right\}$ where $U^{B}: S \rightarrow B$ and $U^{\mathbf{Y}}: S \rightarrow \mathbf{Y}$. Consider the functional (6) written as:

$$
\begin{aligned}
\widetilde{F}\left(U^{B}, U^{\mathbf{Y}}\right)= & \frac{1}{2} \int_{S}\left[\gamma \operatorname{Tr}\left(\nabla U^{B}(s)^{T} \nabla U^{B}(s)\right)\right. \\
& \left.+\operatorname{Tr}\left(\nabla U^{\mathbf{Y}}(s)^{T} \nabla U^{\mathbf{Y}}(s)\right)\right] d s+\alpha \mathcal{D}^{2}(\mu, U) .
\end{aligned}
$$

This representation allows us to define the domain of $U^{\mathbf{Y}}$ as $\mathbb{H}^{1}(S, \mathbf{Y})$ without any boundary condition:

$$
\mathcal{D O} \mathcal{M}_{\mathbf{Y}}:=\left\{U^{\mathbf{Y}} \in \mathbb{H}^{1}(S, \mathbf{Y})\right\} .
$$

The mapping $U^{B}$, on the other hand, must map $S$ onto $B$ such that $\left.U^{B}\right|_{C_{S}}$ : $C_{S} \rightarrow C_{B}:=\partial B$ is a homeomorphism $\left(C_{S}:=\partial S\right.$ is the "frame" of the image). 
This corresponds to treating $U_{B}$ as in a Plateau problem with non-free boundary condition. Following the common wisdom, we need the technical 3-point condition (see [3], p. 235) and set:

$$
\begin{aligned}
& \mathcal{D O} \mathcal{M}_{B}:= \\
& \left\{U^{B} \in \mathbb{H}^{1}(S, B) \cap C^{0}\left(C_{S}, C_{B}\right)\left|U^{B}\right|_{C_{S}}: C_{S} \rightarrow C_{B}\right. \text { is a } \\
& \text { homeomorphism such that } U^{B}\left(s_{i}\right)=\zeta_{i} \text { for } i=1,2,3, \\
& \text { where } s_{1}, s_{2}, s_{3} \text { are three distinct points on } C_{S} \text {, while } \zeta_{1}, \zeta_{2}, \zeta_{3} \\
& \text { are three distinct points on } \left.C_{B} \text { with the same ordering }\right\}
\end{aligned}
$$

Theorem 1. If $\mu$ has a compact support in $E$ then a minimizer of $\widetilde{F}$ is attained in the domain $\mathcal{D O} \mathcal{M}:=\mathcal{D O} \mathcal{M}_{B} \times \mathcal{D O} \mathcal{M}_{\mathbf{Y}}$. Moreover, any minimizer $\bar{U}$ is a minimizer of $F$ (3) as well.

We use a powerful approach that has appeared in the mathematical theory of approximation of functionals via $\Gamma$-convergence. The idea is to approximate the functional (6) with the lack of regularity (due to the metric term $\mathcal{D}(\mu, U)$ ) by a series of different, parameter dependent functionals, that are expected to be more regular. In addition, we expect from convergence of functionals to imply the convergence of minimizers.

Given $\beta>0$, set

$$
D_{\beta}(\mu, \nu)=\sqrt{\beta \ln \left[\int_{E} \frac{\nu(d v)}{\int_{E} e^{-|u-v|^{2} / \beta} \mu(d u)}\right]} .
$$

Lemma 3. $D(\mu, \cdot)$ is the $\Gamma$-limit of $D_{\beta}(\mu, \cdot)$ as $\beta \rightarrow 0$ from above, if considered as a functional of the second argument. That is:

$$
\begin{gathered}
\lim _{\beta \rightarrow 0 ; \beta>0} D_{\beta}(\mu, \nu)=D(\mu, \nu), \\
\liminf _{n \rightarrow \infty} D_{\beta_{n}}\left(\mu, \nu_{n}\right) \geq D(\mu, \nu),
\end{gathered}
$$

where $\beta_{n} \rightarrow 0$ from above and $\nu_{n} \rightarrow \nu$ in $C^{*}$.

We introduce now an implementation of the relaxed distance function $D_{\beta}$ and define a corresponding version of the metric $\mathcal{D}_{\beta}(\mu, U), U \in \mathbb{H}^{1}(S, E)$. This enables us to introduce the relaxation of $\widetilde{F}(6)$ by

$$
\widetilde{F}_{\beta}(U)=\frac{1}{2} \int_{S} \operatorname{Tr}\left(\nabla U(s)^{T} h \nabla U(s)\right) d s+\alpha \mathcal{D}_{\beta}^{2}(\mu, U) .
$$


We set the $\beta$-distance between a mapping $U: S \rightarrow E$ to a measure $\mu$ as

$$
\mathcal{D}_{\beta}(\mu, U):=D_{\beta}\left(\mu, \nu_{U}\right)=\sqrt{\beta \ln \left[\int_{S} \frac{d s}{\int_{E} e^{-|U(s)-z|^{2} / \beta} \mu(d z)}\right]} .
$$

Thus

$$
\mathcal{D}_{\beta}^{2}(\mu, U)=\beta \ln \left[\int_{S} \Xi_{\beta}(U)(s) d s\right],
$$

where

$$
\Xi_{\beta}(U)(s)=\frac{1}{\int_{E} e^{-|U(s)-z|^{2} / \beta} \mu(d z)} .
$$

From Lemma 3 we obtain:

Corollary 1. $\mathcal{D}(\mu, \cdot)$ is the $\Gamma$-limit of $\mathcal{D}_{\beta}(\mu, \cdot)$ as $\beta \rightarrow 0$ from above.

Eventually, we obtain:

Theorem 2. For any $\beta>0$, the functional (9) attains a minimizer $U_{\beta} \in$ $\mathcal{D O} \mathcal{M}$. The sequence of minimizers $\left\{U_{\beta}\right\}$ is bounded in $\mathbb{H}^{1}(S, E)$. Any weak limit $U$ in $\mathbb{H}^{1}$, s.t.

$$
U_{\beta} \stackrel{\beta \rightarrow 0}{\longrightarrow} U \in \mathcal{D O} \mathcal{M}
$$

is a minimizer of $F$ (3) and $\widetilde{F}$, and is a conformal embedding of $S$ into $E$.

The functional $\widetilde{F}_{\beta}$ equipped with the relaxed distance $\mathcal{D}_{\beta}$ is not parameter independent, and its minimizers are not conformal mappings. However, it converges to parameter independent functional $\widetilde{F}$ as $\beta \rightarrow 0$.

\section{Non-parametric Representation}

The parametric model is presented in [5]. We therefore proceed to present the non-parametric representation. Let consider a non-parametric representation of the image $U$. Here we identify $S$ with $B$ and set $U^{B}$ as identity map. Thus, the image is given in terms of a graph $U^{\mathbf{Y}}:=f: B \rightarrow \mathbf{Y}$. Considering the image $U$ in terms of a graph of function $f$ does not allow existence of edges in form of the surface folding. In case of non-parametric formulation we cannot use the majorant area functional $\widetilde{A}(U)$ as defined in (4). The area functional is then given as in (2):

$$
A(U)=\int_{S}|\Gamma(U)(s)| d s=\int_{B} \sqrt{\gamma^{2}+\gamma|\nabla f|^{2}} d b .
$$


We also separate the scale $\gamma$ into $\gamma_{1}$ for the area term and $\gamma_{2}$ for the metric. In order to unify the limits $\gamma_{1} \rightarrow 0$ and $\gamma_{1} \rightarrow \infty$ we normalize the area term by $\frac{1+\sqrt{\gamma_{1}}}{\sqrt{\gamma_{1}}}$. Thus, the corresponding functional is

$$
\mathbf{F}_{\gamma_{1}, \gamma_{2}}^{\beta}(f)=\left(1+\sqrt{\gamma_{1}}\right) \int_{B} \sqrt{\gamma_{1}+|\nabla f|^{2}} d b+\alpha \mathcal{D}_{\beta, \gamma_{2}}^{2}(\mu, f) .
$$

We define now the relaxed metric term $\mathcal{D}_{\beta, \gamma_{2}}(\mu, f)$. For non-parametric formulation we represent the data $\mu(d z)$ as $\mu_{b}(d y) d b$. Under the above assumption, the parameter dependent metric (10) attributes the measure $d b$ to the image $f$, and it is written as

$$
\mathcal{D}_{\beta, \gamma_{2}}^{2}(\mu, f)=\beta \ln \left[\int_{B} \Psi_{\beta, \gamma_{2}}^{f}(b) d b\right]
$$

where

$$
\Psi_{\beta, \gamma_{2}}^{f}(b)=\frac{1}{\int_{\mathbf{Y}} \int_{B} e^{-\left[|f(b)-y|^{2}+\gamma_{2}\left|b-b^{\prime}\right|^{2}\right] / \beta} d b^{\prime} \mu_{b^{\prime}}(d y)} .
$$

In addition, the following analysis is performed for deterministic data, thus $\Psi_{\beta, \gamma_{2}}^{f}(b)$ in (11) is given as

$$
\Psi_{\beta, \gamma_{2}}^{f}(b)=\frac{1}{\int_{B} e^{-\left[\left|f(b)-g\left(b^{\prime}\right)\right|^{2}+\gamma_{2}\left|b-b^{\prime}\right|^{2}\right] / \beta} d b^{\prime}} .
$$

Finally, the corresponding functional is:

$$
\mathbf{F}_{\gamma_{1}, \gamma_{2}}^{\beta}(f)=\left(1+\sqrt{\gamma_{1}}\right) \int_{B} \sqrt{\gamma_{1}+|\nabla f|^{2}} d b+\alpha \beta \ln \left[\int_{B} \Psi_{\beta, \gamma_{2}}^{f}(b) d b\right],
$$

where $\Psi_{\beta, \gamma_{2}}^{f}(b)$ is given as in (12). Its minimum is the function $f$ which solves the boundary value problem

$$
\begin{aligned}
& -\operatorname{div}\left(\frac{\left(1+\sqrt{\gamma_{1}}\right) \nabla f}{\sqrt{\gamma_{1}+|\nabla f|^{2}}}\right)+\frac{2 \alpha \Psi_{\beta, \gamma_{2}}^{f}(b)}{\int_{B} \Psi_{\beta, \gamma_{2}}^{f}(b) d b} f \\
& -\frac{2 \alpha\left(\Psi_{\beta, \gamma_{2}}^{f}(b)\right)^{2}}{\int_{B} \Psi_{\beta, \gamma_{2}}^{f}(b) d b} \int_{B} g\left(b^{\prime}\right) e^{-\left[\left|f(b)-g\left(b^{\prime}\right)\right|^{2}+\gamma_{2}\left|b-b^{\prime}\right|^{2}\right] / \beta} d b^{\prime}=0
\end{aligned}
$$

with Neumann boundary condition 


$$
\frac{\partial f}{\partial \boldsymbol{n}}=0 \quad \text { on } \partial B
$$

Note that the function $G(b)=\frac{\left(1+\sqrt{\gamma_{1}}\right)}{\sqrt{\gamma_{1}+|\nabla f|^{2}}}$ in the first term of the left-hand part of (14) may be considered as edge indicator (penalty) function.

Consider now the $M \times N$ image ( $M$ rows and $N$ columns). Let $B \subset \mathbb{R}^{2}$ be its domain and $g(b), b \in B$ denote its graph. We assume that its desired segmentation is the minimum of relaxed functional (13). We search for a weak solution of the Euler-Lagrange equation (14)-(15).

Due to the robustness and simplicity of implementation of the fixed point algorithm [12, we apply it to solve (14)-(15) in this work. The solution, which is the grey-level image, is the desired segmentation. In order to present the results in a pleasant way, the segmentation contour should be obtained. To achieve this, we threshold the accepted segmentation image by using Otsu's method 8]. Finally, Canny edge detector [2] is applied to the binary image, and achieved segmentation contour is applied to the original image $g$.

In this section we are going to point out the influence of parameters $\beta, \alpha, \gamma_{1}, \gamma_{2}$ on the segmentations results. To demonstrate the performance of the proposed method, we used various textured images [5]. Two examples are illustrated here (Fig. 11). We recall that $\beta$ is the convergence parameter and the minimizer of the
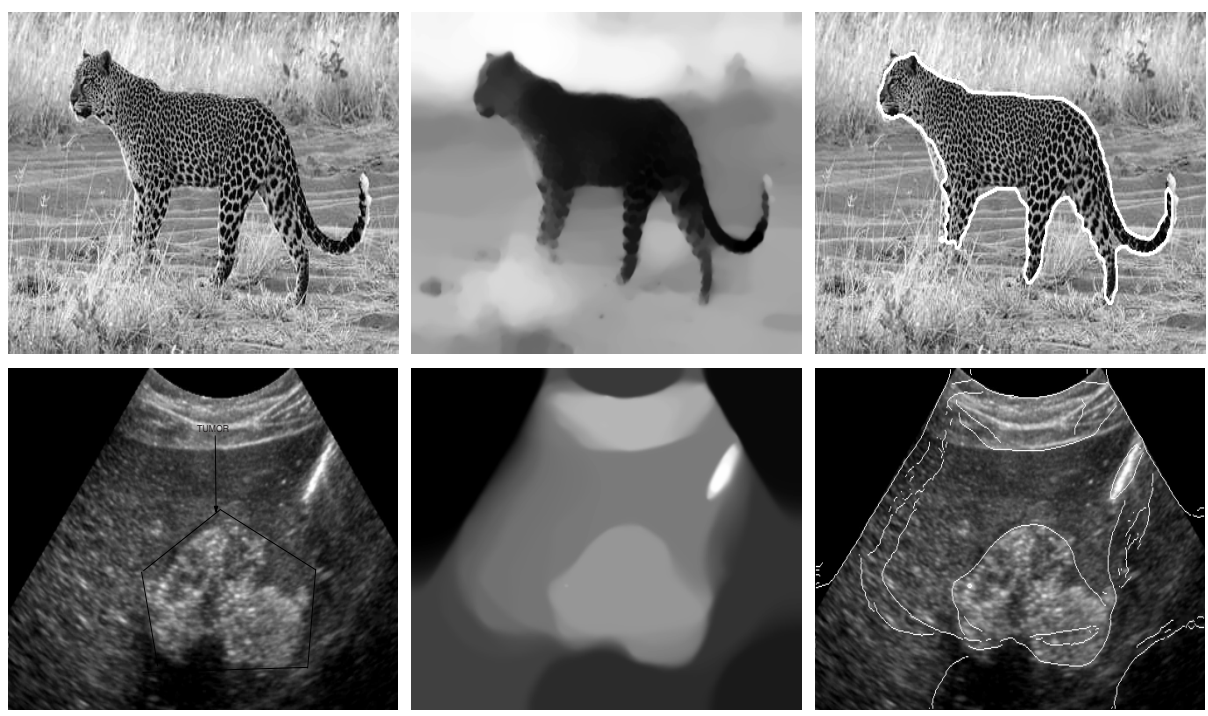

Fig. 1. Leopard (upper row) and ultrasound (lower row) image segmentation using the proposed method. Original images (left), algorithm outputs (middle), outlined segmented images (right). Parameters: $\alpha=185$ (leopard), 50 (ultrasound), $\beta=0.1, \gamma_{1}=0, \gamma_{2}=\infty$. 
original (the limit) functional is achieved when $\beta \rightarrow 0$. We also note that the limit $\beta \rightarrow 0$ of (13) yields

$$
\begin{aligned}
\mathbf{F}_{\gamma_{1}, \gamma_{1}}^{0}(f)= & \left(1+\sqrt{\gamma_{1}}\right) \int_{B} \sqrt{\gamma_{1}+|\nabla f|^{2}} d b+\alpha \sup _{b \in B} \inf _{b^{\prime} \in B}\left[\left|f(b)-g\left(b^{\prime}\right)\right|^{2}\right. \\
& \left.+\gamma_{2}\left|b-b^{\prime}\right|^{2}\right],
\end{aligned}
$$

which is the original functional for deterministic images. Thus, for our calculations, $\beta$ is taken sufficiently small. The parameter $\alpha$, as in case of Mumford-Shah functional, measures the trade-off between a good fit of the solution $f$ to data $g$ and the regularity of the solution $f$.

We define now two limiting cases for the functional (13): $\mathbf{F}_{0, \gamma_{2}}^{\beta}(f)$ and $\mathbf{F}_{\infty, \gamma_{2}}^{\beta}(f)$, when $\gamma_{1} \rightarrow 0$ and $\gamma_{1} \rightarrow \infty$, respectively. As $\gamma_{1}$ tends to 0 , one can obtain

$$
\mathbf{F}_{0, \gamma_{2}}^{\beta}(f)=T V(f)+\alpha \beta \ln \left[\int_{B} \Psi_{\beta, \gamma_{2}}^{f}(b) d b\right],
$$

where $T V(f) \equiv \int_{B}|\nabla f| d b$ is the Total Variation (TV) norm, originally introduced by Rudin et. al. in [10. On the other hand, $\gamma_{1} \rightarrow \infty$ yields

$$
\mathbf{F}_{\infty, \gamma_{2}}^{\beta}(f) \approx H^{1}(f)+\alpha \beta \ln \left[\int_{B} \Psi_{\beta, \gamma_{2}}^{f}(b) d b\right]
$$

up to the constant, where $H^{1}(f) \equiv \int_{B}|\nabla f|^{2} d b$ is Sobolev space norm. We note that the $T V$ norm allows discontinuities in $f$, thus making it superior to the $H^{1}$ regularization in cases where $f$ can have sharp edges. As is shown above, the parameter $\gamma_{1}$ being defined as representing the scale difference between pixel domain and feature fiber, also determines the kind of regularization for function $f$, defining the various regularization norms.

The role of parameter $\gamma_{2}$, which similarly to parameter $\gamma_{1}$ being defined as representing the scale difference between pixel domain and feature fiber, is completely different. Here the case of interest is the limit $\gamma_{2} \rightarrow \infty$. It is easily verified that, as $\gamma_{2} \rightarrow \infty$,

$$
\lim _{\gamma_{2} \rightarrow \infty} \Psi_{\beta, \gamma_{2}}^{f}(b)=C \frac{\gamma_{2}}{\beta} \cdot e^{|f(b)-g(b)|^{2} / \beta},
$$

where $C$ stands for a generic constant. Thus, the limit $\gamma_{2} \rightarrow \infty$ yields

$$
\begin{aligned}
\mathbf{F}_{\gamma_{1}, \infty}^{\beta}(f) & =\lim _{\gamma_{2} \rightarrow \infty} \mathbf{F}_{\gamma_{1}, \gamma_{2}}^{\beta}(f) \approx\left(1+\sqrt{\gamma_{1}}\right) \int_{B} \sqrt{\gamma_{1}+|\nabla f|^{2}} d b \\
& +\alpha \beta \ln \left[\int_{B} e^{|f(b)-g(b)|^{2} / \beta} d b\right]-\alpha \beta \ln \beta
\end{aligned}
$$


up to the constant multiplied by $\beta$. Finally, we note that the limit $\beta \rightarrow 0$ of (16) yields

$$
\mathbf{F}_{\gamma_{1}, \infty}^{0}(f)=\left(1+\sqrt{\gamma_{1}}\right) \int_{B} \sqrt{\gamma_{1}+|\nabla f|^{2}} d b+\alpha \sup _{b \in B}|f(b)-g(b)|^{2} .
$$

The parameter $\gamma_{2}$ determines the local neighborhood of the pixel $b$, which gives the valuable contribution to the final segmentation.

\section{Digital Image Inpainting}

The same functional approach is now applied to the problem of inpainting. As in [1, it does not require any user intervention, once the region to be inpainted has been selected. Consider the 2D discrete grey level image $g$, being defined on the domain $B \backslash D$, where $D$ is the region to be inpainted. Our functional for the two-dimensional image in the non-parametric case accepts the following form:

$$
\begin{aligned}
F_{\gamma_{1}, \gamma_{2}}(f)= & \left(1+\sqrt{\gamma_{1}}\right) \int_{B} \sqrt{\gamma_{1}+|\nabla f|^{2}} d b+\alpha \sup _{b \in B} \inf _{b^{\prime} \in B}\left[\left|f(b)-g\left(b^{\prime}\right)\right|^{2}\right. \\
& \left.+\gamma_{2}\left|b-b^{\prime}\right|^{2}\right] .
\end{aligned}
$$

Let $f$ be a desired inpainting of the image $g$ on the region $D$, be defined on the entire domain $B$. We assume that it should be a minimum of the functional (17). Actually, for the inpainting task the above functional is not applicable: let denote

$$
Q_{\gamma_{2}}^{g}(b)=\inf _{b^{\prime} \in B}\left[\left|f(b)-g\left(b^{\prime}\right)\right|^{2}+\gamma_{2}\left|b-b^{\prime}\right|^{2}\right] .
$$

We claim that, if $g$ is defined everywhere on $B$, then

$$
\lim _{\gamma_{2} \rightarrow \infty} Q_{\gamma_{2}}^{g}(b)=|f(b)-g(b)|^{2} .
$$

If $g$ is not defined in an open set $D$, then $Q_{\gamma_{2}}^{g}(b)$ is replaced by

$$
Q_{\gamma_{2}}^{g}(b)=\inf _{b^{\prime} \in B \backslash D}\left[\left|f(b)-g\left(b^{\prime}\right)\right|^{2}+\gamma_{2}\left|b-b^{\prime}\right|^{2}\right]
$$

and

$$
\lim _{\gamma_{2} \rightarrow \infty} Q_{\gamma_{2}}^{g}(b)=\infty \quad \text { for } \quad b \in D .
$$

Thus, we replace $Q_{\gamma_{2}}^{g}(b)$ by

$$
\tilde{Q}_{\gamma_{2}}^{g}(b)=Q_{\gamma_{2}}^{g}(b)-\gamma_{2} \operatorname{Dis}^{2}(b, B \backslash D),
$$

where $\operatorname{Dis}(b, B \backslash D)$ is the minimal distance between $b$ and $B \backslash D$, and the desired functional is

$$
\begin{aligned}
& \mathbf{F}_{\gamma_{1}, \gamma_{2}}(f)=\left(1+\sqrt{\gamma_{1}}\right) \int_{B} \sqrt{\gamma_{1}+|\nabla f|^{2}} d b \\
& +\alpha \operatorname{ess} \sup _{b \in B} \inf _{b^{\prime} \in B \backslash D}\left[\left|f(b)-g\left(b^{\prime}\right)\right|^{2}+\gamma_{2}\left(\left|b-b^{\prime}\right|^{2}-D i s^{2}(b, B \backslash D)\right)\right] .
\end{aligned}
$$


Let now consider the limit $\gamma_{2} \rightarrow \infty$. We define

$$
\tilde{g}(b):=\left\{\begin{array}{rl}
g(b), & b \in B \backslash D \\
g\left(y_{0}(b)\right), & b \in D
\end{array},\right.
$$

where $y_{0}(b) \in \partial D$ is defined by

$$
\left|y_{0}(b)-b\right| \leq|y-b|, \quad \forall y \in B \backslash D .
$$

Note that $y_{0}(b)$ and thus, $\tilde{g}(b)$ are not defined unambiguously on $D$. According to its definition, the skeleton of $D$ is of zero Lebesgue measure. This fact explains the change from supremum to essential supremum in the definition of metric term of (18). It is also obvious that

$$
\lim _{\gamma_{2} \rightarrow \infty} Q_{\gamma_{2}}^{\tilde{g}}(b)=|f(b)-\tilde{g}(b)|^{2}
$$

for almost any $b$. Thus, the functional (18) has a limit as $\gamma_{2} \rightarrow \infty$, that is

$$
\lim _{\gamma_{2} \rightarrow \infty} \mathbf{F}_{\gamma_{1}, \gamma_{2}}(f)=\left(1+\sqrt{\gamma_{1}}\right) \int_{B} \sqrt{\gamma_{1}+|\nabla f|^{2}} d b+\alpha \operatorname{ess} \sup _{b \in B}|f(b)-\tilde{g}(b)|^{2} .
$$

As in case of image segmentation model, we approximate the functional (18) by relaxed functional with parameter dependent metric using $\Gamma$-convergence technique. The desired relaxed functional is

$$
\mathbf{F}_{\gamma_{1}, \gamma_{2}}^{\beta}(f)=\left(1+\sqrt{\gamma_{1}}\right) \int_{B} \sqrt{\gamma_{1}+|\nabla f|^{2}} d b+\alpha \beta \ln \left[\int_{B} \Psi_{\beta, \gamma_{2}}^{f}(b) d b\right],
$$

where

$$
\Psi_{\beta, \gamma_{2}}^{f}(b)=\frac{1}{\int_{B \backslash D} e^{-\left[\left|f(b)-g\left(b^{\prime}\right)\right|^{2}+\gamma_{2}\left(\left|b-b^{\prime}\right|^{2}-D i s^{2}(b, B \backslash D)\right)\right] / \beta} d b^{\prime}} .
$$

In the same manner, we set the Euler-Lagrange equation of the functional (19) to be

$$
\begin{aligned}
& -\operatorname{div}\left(\frac{\left(1+\sqrt{\gamma_{1}}\right) \nabla f}{\sqrt{\gamma_{1}+|\nabla f|^{2}}}\right)+\frac{2 \alpha \Psi_{\beta, \gamma_{2}}^{f}(b)}{\int_{B} \Psi_{\beta, \gamma_{2}}^{f}(b) d b} f(b) \\
& -\frac{2 \alpha\left(\Psi_{\beta, \gamma_{2}}^{f}(b)\right)^{2}}{\int_{B} \Psi_{\beta, \gamma_{2}}^{f}(b) d b} \int_{B \backslash D} g\left(b^{\prime}\right) e^{-\left[\left|f(b)-g\left(b^{\prime}\right)\right|^{2}+\gamma_{2}\left(\left|b-b^{\prime}\right|^{2}-D i s^{2}(b, B \backslash D)\right)\right] / \beta} d b^{\prime}=0
\end{aligned}
$$

with natural boundary condition

$$
\left.\frac{\partial f}{\partial \boldsymbol{n}}\right|_{\partial B}=0
$$

Fig. 2] shows the results of inpainting a natural image for various $\gamma_{2}$. The user only supplied the "mask" image, using paintbrush-like program. The basic idea 

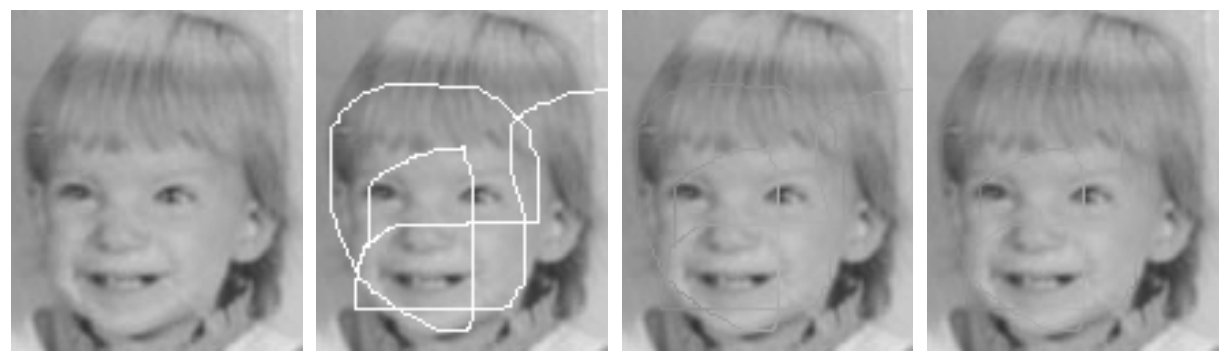

Fig. 2. Output results of inpainting algorithm for real-life image: $\alpha=100000, \gamma_{1}=0$; from left to right: original image, "masked" image, reconstructed image with $\beta=$ $0.07, \gamma_{2}=10$, reconstructed image with $\beta=0.1, \gamma_{2}=100$

is to complete the "masked" region according to minimized modified Hausdorff metric between the corrupted image and its reconstruction.

\section{Discussion and Conclusions}

The present study is a step towards the development of a general framework that can deal with segmentation problems in the context of multi-channel images. The main novelty of this study is the replacement of the metric term of MumfordShah functional by a metric based on Hausdorff distance function. This may be useful in cases of defocusing and of mapping problems. The proposed change allows us to replace the deterministic data by a random one and include also the case of missing data (inpainting).

Since the new metric term, and thus the functional, suffered from lack of regularity, we utilized an approach adopted from mathematical theory of approximation of functionals via $\Gamma$-convergence to overcome this deficiency. However, we should point out that the developed relaxed functional demands extensive computational effort to obtain its minimum. This is the main drawback of our algorithm. An optional solution to this problem is to apply a multi-resolution analysis, by performing the relative computations on higher levels of a Gaussian pyramid and thereby reduce significantly the amount of required computations.

Acknowledgement. This paper is partially supported by the Israel Science Foundation, grant 406/05, founded by the Israel Academy of Sciences and Humanities.

\section{References}

1. M. Bertalmio, G. Sapiro, V. Caselles, and C. Ballester, Image inpainting, Computer Graphics (SIGGRAPH 2000), pp. 417-424, July 2000.

2. J. Canny, A Computational Approach to Edge Detection, IEEE Transactions on PAMI, 8(6): 679-698 (1986). 
3. U. Dierkes, S. Hildebrandt, A. Kuster and O. Wohlrab, Minimal Surfaces I, Springer-Verlag Ser., 295 (1991).

4. R. Kimmel, R. Malladi and N. Sochen, On the geometry of texture, Report LBNL 39640, LBNL, UC-405 UC Berkeley, November 1996.

5. V. Kluzner, G. Wolansky and Y. Y. Zeevi, Minimal surfaces, measure-based metric and image segmentation, CCIT Report No. 605, EE Pub No. 1562, November 2006.

6. J. M. Morel and S. Solimini, Variational Methods in Image Segmentation, Birkhauser, Boston, MA, 1995.

7. D. Mumford and J. Shah, Optimal approximations by piecewise smooth functions and associated variational problems, Comm. Pure Appl. Math., 42: 577-685 (1989).

8. N. Otsu, A Threshold Selection Method from Gray-Level Histograms, IEEE Transactions on Systems, Man, and Cybernetics, 9 (1): 62-66 (1979).

9. T. Richardson and S. Mitter, Approximation, computation and distortion in the variational formulation, In Comm. Geometric-Driven Diffusion in Computer Vision, Ed. B. M. ter Haar Romeny, Kluwer Academic Publishers, 1994.

10. L. I. Rudin, S. Osher and E. Fatemi, Nonlinear total variation based noise removal algorithms, Physica D., 60: 259-268 (1992).

11. N. A. Sochen, R. Kimmel and R. Malladi, A general framework for low-level vision, IEEE Trans. Image Processing, 7: 310-318 (1998).

12. C. Vogel and M. Oman, Iterative methods for total variation denoising, SIAM J. Sci. Statist. Comput., 17(1): 227-238 (1996). 